\title{
Une nouvelle mesure, fondée sur la population, du fardeau économique de la maladie mentale au Canada
}

\author{
K-L Lim, Ph.D. (1); P Jacobs, Ph.D. (2); A Ohinmaa, Ph.D. (3); D Schopflocher, Ph.D. (4); CS Dewa, Ph.D. (5)
}

\section{Résumé}

Nous présentons ici une mesure globale du fardeau économique excédentaire associé à la maladie mentale au Canada, qui tient compte de l'utilisation des ressources médicales et des pertes de productivité imputables aux incapacités de courte et de longue durée, ainsi que de la diminution de la qualité de vie liée à l'état de santé (QVLES), dans la population de personnes souffrant de problèmes de santé mentale, diagnostiqués ou non. L'analyse était fondée sur le cycle 2.1 de l'Enquête sur la santé dans les collectivités canadiennes (2003), réalisée auprès de la population. Nous avons mesuré, à l'égard de tous les sujets, tous les services de santé utilisés, la perte de productivité attribuable à une incapacité de courte ou de longue durée et la qualité de vie liée à l'état de santé, et déterminé la valeur de ces facteurs en dollars; le fardeau économique est la différence, représentée par la valeur en dollars de ces facteurs, entre les populations souffrant ou non de maladies mentales. Le fardeau économique total s'est chiffré à 51 milliards \$ en 2003. Plus de la moitié de ce fardeau était associé à une diminution de la QVLES. La pratique courante est d'inclure dans les évaluations économiques les changements liés à l'utilisation des ressources médicales, la perte de productivité et la diminution de la QVLES.

Mots clés : fardeau économique, coûts de la maladie, santé mentale, qualité de vie

\section{Introduction}

La maladie mentale fait partie d'une catégorie d'affections qui entraînent des effets considérables sur les plans de l'utilisation des ressources médicales, de la perte de productivité et de la souffrance humaine. Deux récents rapports, l'un réalisé par Santé Canada ${ }^{(1)}$ et l'autre par le Sénat ${ }^{(2)}$, ont souligné l'importance du fardeau économique de la maladie mentale au Canada.

Dans son rapport de 2002 intitulé Rapport sur les maladies mentales au Canada ${ }^{(3)}$, Santé Canada a déterminé que la maladie mentale avait des répercussions économiques énormes, sous forme de coûts directs liés à l'utilisation de services de santé financés par l'État (4,7 milliards \$ en 1998) et de coûts indirects liés à la perte de productivité attribuable aux incapacités de courte et de longue durée et aux décès prématurés (3,2 milliards $\$$ ). Selon une étude réalisée en 1997 par Santé Canada ${ }^{(4)}$, à la lumière de plusieurs types de données administratives et de données d'enquête, le fardeau économique (coûts directs et indirects) des maladies mentales au Canada s'élevait à 7,3 milliards \$ en 1993 (ou à 8,4 milliards $\$$ en 1998). En se fondant sur les données de l'étude de Santé Canada de 1997 ainsi que sur certaines questions portant sur la détresse et la dépression posées dans le cadre de l'Enquête nationale sur la santé de la population (ENSP) de 1996 à 1997, Stephens et Joubert $^{(5)}$ ont chiffré à 6,3 milliards \$ les coût directs (incluant, à 278 millions \$, le coût des services de santé non pris en charge par l'État). Les coûts indirects liés à la perte de productivité attribuable aux incapacités de courte et de longue durée et aux décès prématurés associée à la dépression et à la détresse ont été à environ 8,1 milliards \$. Le fardeau estimatif total (recalculé) était de 14,4 milliards \$ en 1998. Dans toutes ces estimations, seuls les services de santé mentale étaient inclus dans le calcul des coûts directs.

Or, la mesure du fardeau économique dépend de la définition donnée au concept. Notre définition du fardeau économique est illustrée dans la figure 1. Comme le montre cette figure, le fardeau économique englobe les coûts tant directs qu'indirects. Selon la définition donnée récemment à ce concept, les pertes de qualité de vie liées à l'état de santé (QVLES), p. ex. l'augmentation de la douleur et de la souffrance, sont une composante essentielle du fardeau économique de la maladie ${ }^{(6-9)}$. Comme ces pertes qui découlent de la QVLES viennent alourdir le fardeau économique global de la maladie, elles sont prises en compte par les plus récentes mesures du fardeau économique de la maladie mentale ${ }^{(10)}$. Les études susmentionnées sur le fardeau économique ne tiennent pas compte des pertes de qualité de vie liées à l'état de santé. De plus, dans les estimations évoquées plus haut, le calcul des coûts directs est fondé non pas sur les coûts excédentaires de tous les services de

Coordonnées des auteurs

1 Département d'économie, Université nationale de Singapour

2 Faculty of Medicine, Université de l'Alberta et Institute of Health Economics, Alberta

3 École de santé publique, Université de l'Alberta et Institute of Health Economics, Alberta

4 Surveillance de la santé, Ministère de la Santé et du Mieux-être de l'Alberta

5 Centre de toxicomanie et de santé mentale et Université de Toronto, Département de psychiatrie, Toronto (Ontario)

Correspondance : Arto Ohinmaa, École de santé publique, Université de I'Alberta, 13-103 Clinical Sciences Building, Edmonton (Alberta), Canada T6G 2G3, Tél.

(780) 492-6535, Courriel : arto.ohinmaa@ualberta.ca 
santé, mais sur les coûts des services de santé mentale. Or, les personnes souffrant de maladie mentale utilisent les deux types de services, d'où l'importance capitale de la question de l'utilisation des services de santé. C'est pourquoi nous avons voulu produire une mesure globale du fardeau économique de la maladie mentale au Canada, ce dont nous rendrons compte ici.

Notre étude présente trois caractéristiques importantes : (1) elle comprend une mesure du fardeau que représentent les personnes souffrant de problèmes de santé mentale non diagnostiqués; (2) les mesures sont fondées sur le concept des coûts et des pertes excédentaires liés à des résultats attribuables à la maladie mentale, et non pas uniquement sur les coûts et les pertes bruts, et (3) elle ne se limite pas aux seuls coûts des services de santé mentale, mais comprend ceux de tous les services de santé.

\section{Méthodologie}

\section{Données}

Les données utilisées aux fins de cette analyse proviennent du Fichier de microdonnées à grande diffusion de l'Enquête sur la santé dans les collectivités canadiennes (ESCC, cycle 2.1), réalisée en $2003^{(11)}$. L'ESCC est une enquête transversale bisannuelle menée par Statistique Canada, qui offre des renseignements sur l'état de santé, l'utilisation du système de santé et les déterminants de la santé des Canadiens vivant dans un logement privé. Des données estimatives sur la population ont été obtenues à l'aide des coefficients de pondération de l'échantillonnage fournis par Statistique Canada.

\section{Population étudiée}

L'étude visait uniquement la population adulte âgée de 20 ans et plus. Nous avons cherché à classer les sujets dans une des trois catégories liées à la santé mentale (Problèmes de santé mentale diagnostiqués, Problèmes de santé mentale non diagnostiqués, Aucun problème de santé mentale) à la lumière des réponses fournies à des questions précises tirées du cycle 2.1 de l'ESCC. Toutefois, comme les questions n’ont pas toutes été posées à tous les participants à l'enquête, nos estimations de la prévalence ont été faites à partir des données groupées applicables aux trois catégories, ainsi qu'il est décrit de manière détaillée ci-après.

Nous avons, dans un module de contenu commun sur les maladies chroniques et à l'égard de chacune des catégories de diagnostic suivantes, demandé aux participants d'indiquer s'ils avaient reçu d'un professionnel de la santé un diagnostic de problème mental majeur. Les sujets ont été classés dans la catégorie "Problèmes de santé mentale diagnostiqués " s'ils avaient répondu par l'affirmative à n'importe laquelle des questions suivantes : "Êtesvous atteint de schizophrénie? »; « Souffrezvous d'un trouble de l'humeur tel que la dépression, un trouble bipolaire, la manie ou la dysthymie? »; ou "Souffrez-vous d'un trouble anxieux tel que la phobie, un trouble obsessionnel-compulsif ou un trouble panique? ».

Nous nous sommes inspirés de la méthode de Starkes et coll. ${ }^{(12)}$, en lui donnant un champ d'application plus vaste, pour les calculs relatifs aux problèmes de santé mentale non diagnostiqués. Nous avons classé dans la catégorie "Problèmes de santé mentale non diagnostiqués" les sujets ne figurant pas dans la catégorie "Problèmes de santé mentale diagnostiqués » s’ils avaient répondu néanmoins à au moins un des critères suivants au cours des douze derniers mois :

a. À la question concernant une autoévaluation de la santé mentale, ils ont déclaré que leur état de santé mentale était piètre.

b. Dans un module concernant les contacts avec des professionnels de la santé mentale, ils ont déclaré avoir consulté en personne ou par téléphone un professionnel de la santé au sujet d'un problème émotionnel ou de santé mentale.

c. Dans un module sur le dépistage de la dépression comprenant de nombreuses questions (tirées du Composite Diag- nostic Interview Schedule) ${ }^{(13,14)}$, ils ont obtenu un résultat indiquant une probabilité d'au moins 0,8 d'être atteints d'une dépression clinique.

d. Dans un module sur les idées ou les tentatives de suicide, ils ont indiqué avoir déjà sérieusement envisagé de s'enlever la vie.

Seuls certains sous-segments de la population étudiée ont reçu les questions du module de contenu optionnel relatives aux sous-critères $b, c$ et $d$. Plus précisément, seuls des sujets de Terre-Neuve-etLabrador, de l'Île-du-Prince-Édouard, du Nouveau-Brunswick, du Québec, de l'Ontario, de l'Alberta et des Territoires-duNord-Ouest - en tout, 77,1 \% de l'échantillon pondéré - se sont fait poser les questions relatives au sous-critère $b$ cidessus; seuls les sujets de Terre-Neuve-etLabrador, de l'Île-du-Prince-Édouard, du Nouveau-Brunswick, de certaines régions de l'Ontario et de la Saskatchewan, de l'Alberta, du Yukon, des Territoires-duNord-Ouest et du Nunavut - en tout, 41,8\% de l'échantillon pondéré - se sont fait poser les questions relatives au souscritère $c$ ci-dessus et seuls les sujets du Nouveau-Brunswick, de certaines régions de l'Ontario, de la Saskatchewan, de l'Alberta, des Territoires-du-Nord-Ouest et du Nunavut - en tout, 32,2 \% de l'échantillon pondéré - se sont fait poser les questions relatives au sous-critère $d$ ci-dessus.

La démarche suivie pour estimer l'importance des trois catégories liées à la santé mentale reposait sur un processus séquentiel. Dans un premier temps, nous avons estimé la prévalence de la catégorie " Problèmes de santé mentale diagnostiqués » à partir de l'ensemble des données obtenues. Nous avons ensuite calculé la proportion de personnes ayant répondu au critère $a$ en vue de les inclure dans la catégorie "Problèmes de santé mentale non diagnostiqués " à partir de l'ensemble des données obtenues en réponse à la question relative à l'auto-évaluation de la santé mentale. Nous avons, à partir des sujets qui se sont fait poser les questions relatives aux critères $a$ et $b$, estimé la proportion de ceux qui répondaient au 
critère $b$, mais pas au critère $a$. De même, à partir des sujets qui se sont fait poser toutes les questions relatives aux critères $a, b$ et $c$, nous avons estimé la proportion de ceux qui répondaient au critère $c$, mais pas au critère $a$ ou $b$. Cette même démarche a été suivie à l'égard de la dernière série de questions, en ce sens que nous avons estimé la proportion de personnes ayant répondu au critère $d$, mais pas au critère $a$, $b$ ou $c$. Nous avons ensuite additionné ces proportions afin d'obtenir une seule estimation de la prévalence de la catégorie "Problèmes de santé mentale non diagnostiqués ».

Cette façon de procéder présente deux caractéristiques importantes. D’abord, elle postule que dans les cas où les questions n'ont pas été posées à tous les sujets, le sous-échantillon de sujets auquel elles ont été posées était néanmoins représentatif. Ensuite, la proportion de personnes répondant successivement à chacun des critères est plus faible, de sorte que les erreurs d'estimation attribuables à un biais d'échantillonnage devraient aussi être successivement plus faibles à chaque étape.

Enfin, nous avons estimé par soustraction le nombre de personnes appartenant à la catégorie "Aucun problème de santé mentale ». Les résultats sont présentés dans le premier rang du tableau 1 .

\section{Mesures des résultats}

Nous avons réalisé toutes les mesures des résultats pour chacun des groupes âgesexe et à l'intérieur des trois catégories liées aux problèmes de santé mentale définies plus haut.

\section{Utilisation}

Nous avons évalué les coûts médicaux en nous fondant sur des données autodéclarées par chaque participant à l'enquête relatives à l'utilisation des services de soins de santé pendant une période d'une année, soit sur le nombre de toutes les consultations d'omnipraticiens (autrement dit, pas uniquement des consultations axées sur la santé mentale), de consultations de spécialistes et de journées d'hospitalisation. Nous avons déterminé l'importance de l'utilisation des services médicaux en nous basant sur la question relative au nombre de fois (en excluant les séjours à l'hôpital) où la personne avait vu ou consulté par téléphone le professionnel de la santé (omnipraticien et spécialiste) au sujet de son problème de santé physique, émotionnelle ou mentale au cours de la dernière année. Nous avons déterminé l'ampleur de l'utilisation des ressources des hôpitaux à partir des questions concernant le nombre de nuits passées dans un établissement. Nos estimations portaient sur chaque groupe âge-sexe et sur les trois catégories liées à la santé mentale.

\section{Coûts unitaires}

Nous avons établi les coûts unitaires des services pour les groupes âge-sexe à partir des données statistiques provinciales fournies par le ministère de la Santé et du Mieux-être de l'Alberta pour l'année budgétaire 2000 à $2001^{(15)}$, en rajustant les données pour tenir compte, au besoin, de l'inflation et des honoraires par niveau de service à l'échelle nationale. Le ministère de la Santé et du Mieux-être de l'Alberta tient une base de données sur les demandes de paiement des médecins qui comprend généralement plus de 30 millions d'enregistrements par année. Pour chaque demande de paiement, la base de données rend compte du lieu où le service est offert et du type de service offert ainsi que de la spécialisation du médecin. Le coût moyen par consultation d'un omnipraticien comprenait tant les honoraires des médecins que les coûts des analyses en laboratoire. Les consultations de spécialistes englobaient

\section{TABLEAU 1}

Valeurs unitaires des résultats selon l'état de santé mentale au Canada (2003) chez les personnes de 20 ans et plus

\begin{tabular}{|c|c|c|c|c|}
\hline & $\begin{array}{l}\text { Aucun } \\
\text { problème } \\
\text { de santé } \\
\text { mentale }\end{array}$ & $\begin{array}{l}\text { Problèmes de } \\
\text { santé mentale } \\
\text { diagnostiqués }\end{array}$ & $\begin{array}{l}\text { Problèmes de } \\
\text { santé mentale } \\
\text { non } \\
\text { diagnostiqués }\end{array}$ & Population \\
\hline Fréquence & 23261558 & 1877163 & 1605238 & 26743959 \\
\hline Pourcentage du total & $87,0 \%$ & $7,0 \%$ & $6,0 \%$ & $100,0 \%$ \\
\hline \multicolumn{5}{|l|}{ Hommes } \\
\hline 20 à 34 ans & 3179784 & 150433 & 215549 & \\
\hline 35 à 49 ans & 3822703 & 235545 & 246683 & \\
\hline 50 à 64 ans & 2727415 & 195830 & 120105 & \\
\hline 65 ans et plus & 1659625 & 71528 & 40779 & \\
\hline \multicolumn{5}{|l|}{ Femmes } \\
\hline 20 à 34 ans & 3154711 & 302591 & 332724 & \\
\hline 35 à 49 ans & 3840586 & 433365 & 396629 & \\
\hline 50 à 64 ans & 2751214 & 330160 & 190324 & \\
\hline 65 ans et plus & 2125520 & 157711 & 62445 & \\
\hline \multicolumn{5}{|l|}{$\begin{array}{l}\text { Valeurs par personnes } \\
\text { (moyennes pondérées) }\end{array}$} \\
\hline $\begin{array}{l}\text { Consultation } \\
\text { d'omnipraticiens }\end{array}$ & 2,9 & 6,7 & 4,8 & \\
\hline Consultations de spécialistes & 0,7 & 2,5 & 1,7 & \\
\hline Jours d'hospitalisation & 0,5 & 2,2 & 1,2 & \\
\hline Indice de l'état de santé & 0,91 & 0,71 & 0,8 & \\
\hline $\begin{array}{l}\text { Pourcentage ne travaillant } \\
\text { pas (perte de travail de } \\
\text { longue durée) }\end{array}$ & 0,33 & 0,46 & 0,26 & \\
\hline $\begin{array}{l}\text { Jours d'incapacité par année } \\
\text { (perte de travail de courte } \\
\text { durée) }\end{array}$ & 10 & 33 & 27 & \\
\hline
\end{tabular}


aussi bien les consultations en cabinet que les consultations à l'externe en milieu hospitalier (y compris les consultations en salle d'urgence). Le coût moyen par consultation d'un spécialiste en cabinet comprenait aussi bien les honoraires médicaux que le coût des services diagnostiques. Dans le cas des consultations d'un spécialiste à l'externe en milieu hospitalier, le coût des services hospitaliers de consultations externes (fondés sur le coût provincial par consultation déterminé par l'Alberta Ambulatory Care Classification System) a été ajouté aux honoraires médicaux. Le coût des séjours à l'hôpital comprenait aussi bien le coût des services offerts par l'établissement que les honoraires des médecins. Nous nous sommes fondés sur le coût quotidien provincial de 809 \$ (780 \$ en 2000 à 2001, somme rajustée par un facteur de $6,5 \%$ pour tenir compte du changement de l'Indice des prix à la consommation observé jusqu'en 2003 à 2004) pour une journée d'hospitalisation type, auquel nous avons ajouté les honoraires médicaux moyens par journée d'hospitalisation. Les honoraires médicaux pour l'Alberta ont été rajustés à la baisse de $18 \%$, les honoraires par niveau de service en Alberta étant de $18 \%$ supérieurs à la moyenne nationale ${ }^{(16)}$. Les coûts unitaires des services selon les groupes âge-sexe ont été appliqués à chaque unité de service. Le coût total des soins médicaux par personne représente la somme des coûts des trois types de services.

\section{Perte de travail - mesure et évaluation}

La perte de travail en raison d'une incapacité de longue durée est calculée à partir des données sur le chômage de la dernière année. Nous avons déterminé la proportion de sujets dans chaque groupe âge-sexe n'ayant pas travaillé au cours de l'année en regroupant les réponses à la question de l'ESCC sur le nombre de semaines où la personne a travaillé à un emploi ou à une entreprise au cours de la dernière année (y compris les semaines de congé payé, de congé de maternité payé ou de congé de maladie payé). Nous avons calculé la perte de travail en raison d'une incapacité de longue durée (chômage) en
FIGURE 1

\section{Classification des composantes du fardeau économique}

\begin{tabular}{|l|l|l|l|}
\hline \multirow{2}{*}{ Ressources en santé } & \multicolumn{2}{|l|}{$\begin{array}{l}\text { Coûts directs (assumés } \\
\text { par l'État) }\end{array}$} & \multicolumn{2}{|l|}{$\begin{array}{l}\text { Coûts assumés par les } \\
\text { individus et les sociétés } \\
\text { d'assurance }\end{array}$} \\
\cline { 2 - 4 } Perte de productivité & $\begin{array}{l}\text { Incapacité de } \\
\text { courte durée }\end{array}$ & \multicolumn{1}{|l|}{$\begin{array}{l}\text { Incapacité de } \\
\text { longue durée }\end{array}$} & \multicolumn{1}{c}{$\begin{array}{l}\text { Coût de la } \\
\text { mortalité } \\
\text { prématurée }\end{array}$} \\
\cline { 2 - 4 } Résultats en santé & $\begin{array}{l}\text { Pertes sur le plan de la qualité de vie } \\
\text { liée à l'état de santé, découlant de la } \\
\text { morbidité }\end{array}$ & \\
\hline
\end{tabular}

FIGURE 2

Coût par personne selon la caractéristique de la population (\$ CA)

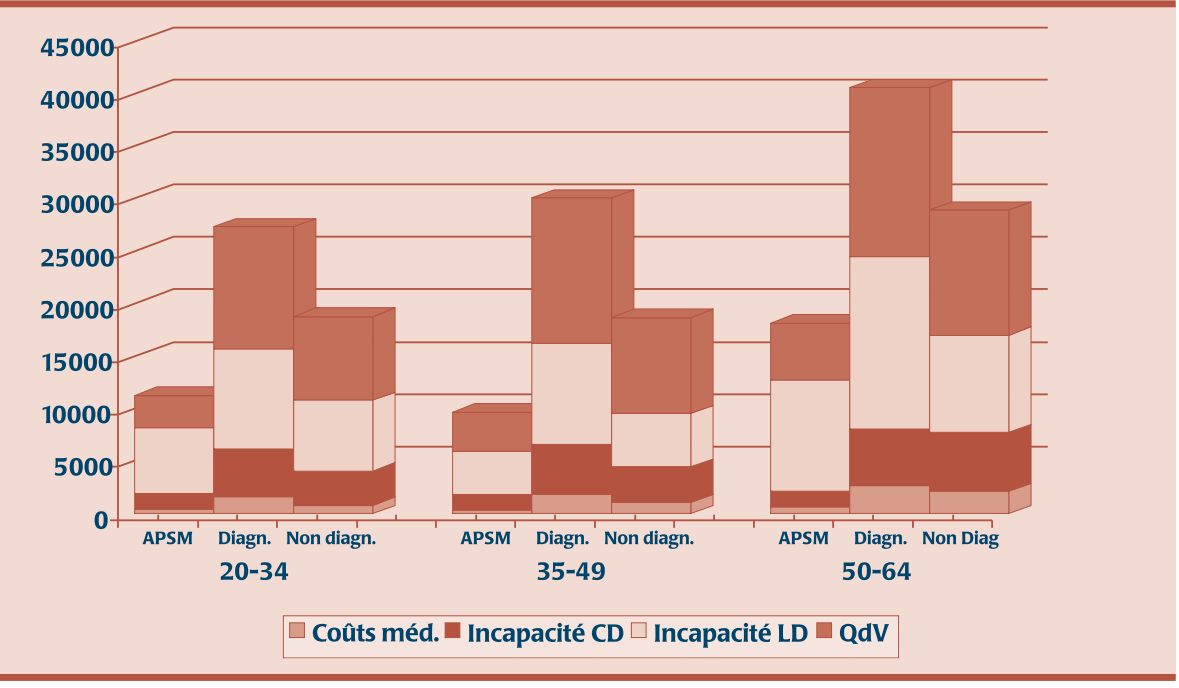

ASPM $=$ aucun problème de santé mentale, Diagn. $=$ problèmes de santé mentale diagnostiqués, Non diagn = problèmes de santé mentale non diagnositiqués, Coûts méd. = coûts médicaux directs, Incapacité $\mathrm{CD}=$ incapacité de courte durée, Incapacité $\mathrm{LD}=$ incapacité de longue durée, $\mathrm{QdV}=$ qualité de vie.

TABLEAU 2

Fardeau économique de la maladie mentale, Canada, 2003

\begin{tabular}{|c|c|c|c|c|}
\hline Élement & $\begin{array}{c}\text { Problèmes } \\
\text { diagnostiqués } \\
\text { (millions \$CA) }\end{array}$ & $\begin{array}{l}\text { Problèmes non } \\
\text { diagnostiqués } \\
\text { (millions \$CA) }\end{array}$ & $\begin{array}{l}\text { Fardeau total } \\
\text { (millions \$ CA) }\end{array}$ & $\begin{array}{l}\text { Pourcentage } \\
\text { du total }\end{array}$ \\
\hline Coûts médicaux directs & 3518 & 1447 & 4965 & 9,8 \\
\hline $\begin{array}{l}\text { Valeur en dollars de la perte } \\
\text { de travail (de longue durée) }\end{array}$ & 8386 & 68 & 8454 & 16,6 \\
\hline $\begin{array}{l}\text { Valeur en dollars de la perte } \\
\text { de travail (de courte durée) }\end{array}$ & 5724 & 3551 & 9275 & 18,2 \\
\hline $\begin{array}{l}\text { Valeur en dollars de la perte } \\
\text { d'utilité en santé }\end{array}$ & 18750 & 9403 & 28153 & 55,4 \\
\hline Fardeau total & 36378 & 14469 & 50847 & $100,0 \%$ \\
\hline
\end{tabular}


fonction des données sur les gains annuels moyens selon le sexe, fournies par Statistique Canada [www.statcan.ca, tableau 202-0102], pour l'année 2003. Les gains annuels moyens, en 2003, étaient de 25300 \$ pour les femmes et de $40200 \$$ pour les hommes.

Nous avons aussi calculé la perte de travail en raison d'une incapacité de courte durée (absentéisme) à partir des réponses aux questions sur le nombre de jours où la personne a dû garder le lit pendant toute la journée ou presque (y compris les jours d'hospitalisation) au cours des 14 jours précédant l'enquête. Nous avons multiplié par 5/7 le nombre moyen de jours d'incapacité applicable à la période de deux semaines pour chaque groupe âgesexe en postulant que les jours d'incapacité étaient également répartis entre les jours de semaine et les fins de semaine. Nous avons multiplié ce nombre par 26 semaines afin d'obtenir le chiffre annualisé, que nous avons multiplié par les gains quotidiens obtenus de Statistique Canada. Les gains hebdomadaires moyens, pour les travailleurs à plein temps et à temps partiel de toutes les industries, s'élevaient à $690,57 \$$ en 2003. Nous avons divisé ce chiffre par 5 jours ouvrables pour obtenir un chiffre quotidien de 138,11 \$, somme que nous avons ensuite appliqué aux hommes et aux femmes de chaque groupe âge-sexe.

\section{Utilité en santé - mesure et évaluation}

Nous avons déterminé la qualité de vie liée à l'état de santé (QVLES) de chaque personne à l'aide de l'indice de l'état de santé HUI3 (Health Utilities Index Mark 3). Le HUI3 est un outil multidimensionnel de mesure de la QVLES, fondé sur des préférences, qui attribue une valeur à différents états de santé à l'aide d'une fonction d'utilité multiplicative ${ }^{(17,18)}$. Selon le HUI3, l'état de santé est défini en fonction de huit dimensions - vue, ouie, parole, mobilité, dextérité, émotion, cognition et douleur. Chacune des dimensions comporte différents niveaux, et les scores de préférence fondés sur l'utilité sont cumulés multiplicativement, ce qui donne un score ou un indice global de l'état de santé qui mesure la qualité de vie associée à l'état de santé de chaque individu. Les scores globaux relatifs à l'indice de l'état de santé (HUI3), dans le cycle 2.1 de l'ESCC, varient de $-0,36$ à 1,0 , où $-0,36$ représente le pire état de santé possible, 0,0 , le décès et 1,0 , la parfaite santé. Une année en parfaite santé équivaut à une année de survie ajustée pour la qualité de vie (QALY). Selon Drummond ${ }^{(7)}$, des écarts de 0,03 entre les scores globaux d'utilité sont considérées comme cliniquement significatifs.

Dans l'ESCC, l'état de santé fait l'objet d'un module de contenu optionnel questionnaire qui n’a été choisi que par cinq provinces : Terre-Neuve-et-Labrador, l'Îledu-Prince-Édouard, la Nouvelle-Écosse, le Nouveau-Brunswick et le Québec. Ainsi, l'analyse a fait appel aux coefficients de pondération de l'échantillonnage des cinq provinces afin d'appliquer aux trois catégories relatives à la santé mentale les scores relatifs à l'indice HUI3 des populations. Afin d'obtenir une évaluation en dollars des diminutions de l'utilité en santé, nous avons multiplié le nombre de QALY perdues par $50000 \$$, chiffre couramment utilisé dans l'élaboration de politiques $^{(19)}$.

\section{Méthodes d'analyse}

Notre définition du fardeau économique met l'accent sur les coûts et pertes excédentaires liés à des conséquences de la maladie mentale. Elle tient compte (1) de l'excédent de coûts liés à l'utilisation de services médicaux découlant d'une maladie mentale, (2) de l'excédent de perte de travail, tant à long terme (chômage) qu'à court terme (absentéisme), découlant du trouble et (3) de l'excédent de perte d'utilité en santé attribuable à la maladie mentale. Les trois composantes sont exprimées sous forme d'écarts, dans ces mesures, entre les populations qui présentent des problèmes de santé mentale (diagnostiqués et non diagnostiqués) et la population exempte de problèmes de santé mentale.
Nous avons estimé les coûts médicaux excédentaires ou nets imputables à la maladie mentale de la façon indiquée cidessus pour chaque groupe âge-sexe et nous les avons totalisés afin d'obtenir les coûts excédentaires totaux applicables à chaque catégorie liée à la santé mentale. Dans le cas de l'utilité en santé, les personnes exemptes de maladies mentales ont généralement des scores relatifs à l'indice de l'état de santé HUI3 plus élevés que les personnes atteintes de maladies mentales, diagnostiqués et non diagnostiqués (tableau 1). L'écart entre les scores nous indique les pertes ou les diminutions de l'utilité en santé, attribuables au trouble mental. Nous avons, en commençant par la catégorie des problèmes diagnostiqués, multiplié les pertes d'utilité en santé pour chaque groupe âge-sexe par le nombre de personnes à l'intérieur de chaque sousgroupe afin d'obtenir le nombre de QALY perdues pour chaque sous-groupe. Nous avons ensuite totalisé les QALY perdues pour tous les sous-groupes âge-sexe afin d'obtenir le nombre total de QALY perdues pour la catégorie des problèmes diagnostiqués. Nous avons suivi la même démarche pour la catégorie des problèmes non diagnostiqués. Nous avons estimé l'excédent de perte de revenu attribuable à une incapacité de longue durée (jours de travail perdus en raison du chômage) au cours de la dernière année et à une incapacité de courte durée (jours de travail perdus annualisés en raison de l'absentéisme) d'une façon analogue et avons ajouté le chiffre obtenu aux coûts évoqués plus haut afin d'obtenir le fardeau économique total des groupes de population souffrant de problèmes de santé mentale diagnostiqués et non diagnostiqués au Canada en 2003.

\section{Résultats}

Le tableau 1 indique la population adulte canadienne répartie par composante. Dans l'ensemble de la population, $7 \%$, ou près de 1,9 million de personnes, ont reçu un diagnostic de maladie mentale et $6 \%$, ou 1,6 million de personnes, souffrent d'une maladie mentale non diagnostiquée. La plus forte proportion de personnes atteintes 
de maladie mentale, tant chez les hommes que chez les femmes, font partie du groupe d'âge de 35 à 49 ans, le taux de prévalence étant plus élevé (de 1,5 à 2 fois plus) chez les femmes, dans tous les groupes d'âge, tant pour les problèmes diagnostiqués que pour les problèmes non diagnostiqués.

Le taux d'utilisation de tous les services de soins de santé culmine chez les personnes souffrant d'une maladie mentale diagnostiquée, et il est à son plus bas chez les personnes exemptes de maladie mentale, la catégorie des personnes souffrant d'une maladie non diagnostiquée se situant entre les deux. La même remarque vaut pour l'utilité en santé et l'absentéisme. L'emploi est plus faible chez les personnes souffrant d'un problème diagnostiqué, mais les personnes souffrant d'une maladie non diagnostiquée sont plus nombreuses à travailler que les personnes exemptes de maladie mentale. La valeur en dollars de ces estimations est comparable aux estimations relatives aux problèmes physiques, comme le montre la figure 2 . Les coûts médicaux moyens par habitant s'élevaient à $643 \$$ pour les personnes exemptes de maladie mentale, à 2515 \$ et à 1442 \$, respectivement pour les personnes atteintes d'une maladie mentale diagnostiquée et non diagnostiquée.

Sur le plan des coûts par habitant, la valeur des jours de travail perdus (chômage) est la principale composante, ce qui s'explique par le coût unitaire élevé d'une perte d'emploi annuelle (une moyenne de 32750 \$ par personne).

Le fardeau économique excédentaire de la maladie mentale chez les personnes de plus de 20 ans est indiqué dans le tableau 2. Le fardeau total en 2003 se chiffrait à environ 51 milliards \$, près de $30 \%$ des coûts étant occasionnés par la population atteinte de maladies mentales non diagnostiquées. La perte d'utilité en santé, évaluée à 50000 \$ par année de survie ajustée pour la qualité de vie (QALY), était de loin l'effet le plus important, puisqu'elle représentait plus de $50 \%$ du fardeau total, soit environ 28 milliards \$. La valeur des jours de travail perdus en raison de l'absentéisme (incapacité de courte durée) était environ $10 \%$ supérieure à la valeur des jours de travail perdus en raison du chômage (incapacité de longue durée). Les deux comptent pour à peu près $35 \%$ du fardeau. Les dépenses médicales représentaient moins de $10 \%$ du fardeau total.

\section{Analyse}

Nous avons établi une mesure globale du fardeau économique excédentaire associé aux personnes de 20 ans et plus, atteintes de maladie mentale au Canada en 2003. Selon notre estimation, le fardeau total s'élevait à 51 milliards \$. Les principales composantes sont indiquées dans le tableau 2, la perte d'utilité en santé représentant plus de la moitié du fardeau total.

D'un point de vue conceptuel, nos résultats et notre analyse diffèrent de ceux de Santé Canada $^{3}$ et de Stevens et Joubert (SJ) ${ }^{(5)}$. L'analyse de Santé Canada mesure les coûts directs des soins de santé en se fondant sur les services de santé mentale précisément alors que la nôtre tient compte de tous les services de soins de santé (utilisés par les personnes souffrant de maladie mentale), toutes causes confondues. Nous n'avons cependant pas pu utiliser la démarche retenue par Santé Canada afin de déterminer le nombre de personnes ayant reçu des services. Santé Canada a réalisé son estimation à l'aide d'une démarche descendante, selon laquelle les coûts ont été imputés à la santé mentale (et à d'autres problèmes de santé) selon le mode d'utilisation des services, pas selon les types de patients. Seuls les services spécifiquement marqués d'un code relatif à la santé mentale (dans les facturations et les résumés) ont été inclus dans la démarche de Santé Canada. De plus, nous n'avons tenu compte que des services hospitaliers et médicaux, alors que Santé Canada a aussi pris en compte les médicaments. Les services communautaires de santé mentale étaient exclus tant de notre étude que de celle de Santé Canada et celle de Stevens et Joubert. Ces deux auteurs se sont servis des estimations de Santé Canada pour déterminer les coûts directs, mais ils ont fait appel à une démarche analogue à la nôtre pour établir les coûts indirects (soit les coûts excédentaires fondés sur les données de l’ESCC).
Dans leur définition de la maladie mentale, Stevens et Joubert incluent la dépression et la détresse, alors que la nôtre repose sur la maladie mentale diagnostiquée et non diagnostiquée ${ }^{(5)}$. Cependant, leurs définitions des coûts des pertes liés à des incapacités de courte et de longue durée diffèrent des nôtres. En effet, notre définition des pertes de courte durée ne comprend que le temps perdu associé au travail alors que Stevens et Joubert ont inclus dans leur définition le temps perdu associé à toutes les activités. Notre évaluation des coûts des pertes de longue durée correspond à la valeur de l'écart, sur le plan de l'emploi, entre les personnes atteintes d'une maladie mentale et celles qui en sont exemptes. Stevens et Joubert n'ont inclus que les jours d'inactivité dans le temps net de chômage. Étant donné que nous avons retenu comme mesure de la perte de travail le coût total associé à la perte d'emploi au cours d'une seule année, notre chiffre est bien plus élevé que celui de Stevens et Joubert.

Comme le soulignent Stevens et Joubert ${ }^{(5)}$, l'un des grands avantages de notre démarche, c'est le lien établi entre les ressources utilisées et les personnes. Ainsi, le fardeau global peut être attribué à divers facteurs de risque précis, comme l'âge, le sexe, les indicateurs socio-économiques, conformément à une approche axée sur la santé de la population. Notre démarche comporte un second avantage, qui découle du premier, en ce sens qu'elle nous permet d'estimer l'utilisation additionnelle nette de toutes les ressources médicales, même les ressources non spécifiquement consacrées à la santé mentale. Il arrive en effet que des personnes atteintes de maladie mentale utilisent davantage les services généraux de santé, comme le fera ressortir notre analyse.

Notre approche a aussi la particularité d'inclure dans l'estimation des coûts la population atteinte de maladies mentales non diagnostiquées (ce qui représente environ 1,6 million de personnes). Plus du quart du fardeau total a été attribué à cette population, soit environ $30 \%$ des coûts médicaux directs. Sareen et coll. ${ }^{(20)}$ ont montré, à l'aide du supplément sur la santé mentale de l'Enquête sur la santé en 
Ontario, qu'outre les critères explicitement prévus par un système de diagnostic reconnu, l'autoperception de besoins de traitement en santé mentale offre de précieux éléments d'information lorsqu'il s'agit d'estimer le nombre de personnes ayant besoin de services de santé mentale au sein de la population et de concevoir des stratégies de santé publique permettant de réduire le fardeau de la maladie mentale au Canada.

Il importe de préciser plusieurs postulats importants que nous avons retenus, puisque les résultats dépendent de l'approche choisie. D'abord, l'effet de l'utilité en santé dépend de la valeur attribuée à une QALY. Il aurait été considérable même si l'évaluation de la QALY avait été très prudente, soit de 20000 \$, comme l'a laissé entendre Laupacis $^{(21)}$. Dans ce cas, le fardeau de la QVLES dépasserait malgré tout la somme de 11 milliards $\$$, et correspondrait à près de $35 \%$ du fardeau total. La valeur de 50000 \$, attribuée à la perte d'une QALY, est la plus couramment utilisée, mais elle demeure arbitraire. Si elle était de 10000 \$ inférieure ou supérieure, l'écart par personne diminuerait ou augmenterait d'environ 1500 \$. Quoi qu'il en soit, il est clair que la QVLES représente la principale composante du fardeau économique de la maladie mentale, et que l'on ne peut en faire abstraction.

Deuxièmement, le calcul de la perte annuelle associée aux personnes qui sont sans travail pendant une période prolongée dépend de la perspective de l'étude. D’après la méthodologie retenue dans la présente étude, la perte de valeur équivaut aux revenus perdus pour chaque année « additionnelle » de travail perdu. Il existe une autre approche, celle fondée sur le " coût de friction": si le travailleur est remplacé par un autre qui, autrement, n'aurait pas été employé, il y a un contrepoids à la perte de travail. La valeur pour la société du travail perdu est égale à la perte de revenu subie par le travailleur invalide moins les gains obtenus par le travailleur qui l'a remplacé. Selon une méthode utilisée, le coût de friction estimatif était environ moitié moindre que le coût obtenu à l'aide de la méthode actuelle $^{(22)}$. Si c'était le cas, les pertes de longue durée se rapprocheraient davantage de 9 milliards \$ que de 16 milliards \$, et le fardeau total diminuerait d'autant.

Notre approche, fondée sur une enquête menée dans la communauté, présente plusieurs limites qui se répercuteront également sur notre estimation totale. D'abord les estimations de l'utilisation reposent sur un rappel individuel, qui n'est pas aussi fiable qu'un registre de consultations d'un professionnel de la santé. Toutefois, selon une analyse antérieure fondée sur ces données, les coûts globaux estimés à l'aide de cette méthode rejoignent ceux établis au moyen d'autres méthodes $^{(15)}$.

Une deuxième limite de notre démarche a trait à l'utilisation des coûts unitaires. Nos coûts renvoient aux coûts des soins de santé généraux, pas des services de santé mentale. Les coûts hospitaliers quotidiens, en particulier, sont plus faibles dans le cas des hospitalisations liées à des problèmes psychiatriques que dans le cas des hospitalisations liées à des problèmes généraux. En 2004, le coût des services de santé mentale en milieu hospitalier s'élevaient à environ 600 \$ par jour, alors que d'après notre estimation, le coût des services généraux de santé se chiffraient à 800 \$. Notre calcul des coûts hospitaliers est quelque peu plus élevé, même si certaines de ces hospitalisations concernent des soins non liés à la santé mentale et que leur coût devrait être calculé à un tarif plus élevé. Nous ne possédons aucune information sur le pourcentage que cela représente.

Notre démarche présente une troisième limite, qui est connexe, à savoir que nous ne tenons pas compte explicitement de la contribution des affections physiques concomitantes aux effets de la maladie mentale sur la productivité. Si l'on se fie à la littérature, la présence concomitante d'une affection physique et d'une affection mentale a des répercussions plus grandes sur l'incapacité(23,24). Il serait très difficile, à l'aide de notre méthode d'estimation des coûts liés aux cas non diagnostiqués, d'évaluer la contribution nette des comorbidités.

Une dernière limite a trait aux personnes visées dans l'ESCC. Comme elle a été réalisée dans les ménages, cette enquête n'a pas tenu compte des personnes atteintes d'une maladie mentale qui sont placées en établissement de manière permanente. D’après des estimations obtenues de l'ICIS, en 2003 à 2004, on a dénombré 403 lits affectés à des soins psychiatriques de longue durée dans les hôpitaux psychiatriques spécialisés de l'Ontario ${ }^{(25)}$. Si l'on suppose qu'à l'échelle du Canada, le ratio lits-population était le même qu'en Ontario, on dénombrerait 1039 patients résidants d'établissements de soins de longue durée au Canada. À 365 jours par patient, le nombre total de jours-lits serait de 379000 . À un coût quotidien très approximatif (applicable à l'Ontario) se situant entre 300 et 400 \$ pour les personnes souffrant de maladie mentale placées en établissement [Source : Alberta Mental Health Board] (de 109500 \$ à 146000 \$ par personne par an), le coût lié à ces personnes oscillerait entre 113 et 151 millions \$. Si ce calcul est exact, les coûts du placement en établissement viendraient augmenter d'environ 3 \% nos calculs de coûts directs.

Outre les erreurs d'estimation et d'échantillonnage, notre enquête a exclu du calcul des coûts indirects les écarts liés à la mortalité. Même si les taux de mortalité sont sans doute plus élevés dans la population atteinte de maladie mentale, ils ne seraient probablement pas très différents d'un groupe à l'autre. Nous avons exclu du calcul des coûts directs les services dispensés par les professionnels de la santé mentale, autres que des médecins, comme les travailleurs sociaux et les psychologues. Cette composante viendrait ajouter $4 \%$ à notre estimation $\left(\mathrm{SJ}^{(5)}\right)$. La plus importante de ces omissions concerne le coût des médicaments prescrits à des patients non hospitalisés, lequel n'a pu être calculé à partir de l’ESCC. Selon Santé Canada ${ }^{(3)}$, les coûts estimatifs des médicaments liés à la santé mentale représenteraient $22 \%$ des 
coûts totaux des soins de santé, ce qui laisse supposer que les coûts totaux directs, si on y ajoute les médicaments, seraient d'environ 6 milliards \$, par rapport à une somme d'environ 5 milliards $\$$ si on les exclut.

Pour conclure, le fardeau économique des personnes atteintes de maladie mentale, tel que nous l'avons mesuré, est énorme. Les pertes associées à la QVLES, normalement exclues des estimations, sont considérables, dans cette évaluation. Bien qu'il ne s'agisse pas de coûts (autrement dit, de composantes liées à l'utilisation de ressources), tels qu'ils sont généralement définis, ces pertes occasionnent de la souffrance, et les personnes sont disposées à payer afin de réduire ce fardeau. Autrement dit, elles ont une valeur économique. Toutefois, il importe de souligner qu'il existe diverses façons de définir et de mesurer le fardeau économique, et la façon qui s'impose dépend de l'objet de l'étude. Enfin, les données se sont considérablement améliorées ces dernières années, mais bien que notre étude se rapproche d'une estimation globale, il faudrait obtenir de meilleures données concernant les soins offerts en établissement, les soins communautaires et les médicaments, afin de fournir des estimations globales des coûts qui seront utiles aux décideurs.

\section{Références}

1. Santé Canada. Rapport sur les maladies mentales au Canada. Ottawa : Santé Canada, 2002. No de catalogue : 0-662-877 45-4.

2. Comité sénatorial permanent des affaires sociales, des sciences et de la technologie (présidé par l'honorable Michael J. L. Kirby). Rapport provisoire sur la santé mentale, la maladie mentale et la toxicomanie: Rapports 1, 2 et 3. Ottawa : Sénat du Canada, novembre 2004.

3. Santé Canada. Le fardeau économique de la maladie au Canada, 1998. Ottawa : Santé Canada, 2002. No de cat. : H21-136/1998F.

4. Moore R, Mao Y, Zhang J, Clarke K, Laboratoire de lutte contre la maladie. Le fardeau économique de la maladie au Canada, 1993. Ottawa : Santé Canada, 1997. No de cat. : H21-136/1993E.
5. Stephens $\mathrm{T}$, Joubert $\mathrm{N}$. Le fardeau économique des problèmes de santé mentale au Canada. Maladies chroniques au Canada 2001;22(1):1-10.

6. Phillips C. Health Economics. Londres : BMJ Books, 2005.

7. Drummond M. Introducing economic and quality of life measurements into clinical studies. Ann Med 2001;333:344-349.

8. Guyati G, Feeny D, Patrick D. Measuring health-related quality of life. Ann Intern Med 1993;118:622-729.

9. Schultz SE, Kopec JA. Impact of chronic conditions. Health Rep 2003;14(4):41-53.

10. Sainsbury Centre for Mental Health. The Economic and Social Costs of Mental Illness. Londres : The Sainsbury Centre, 2003.

11. Statistique Canada. Enquête sur la santé dans les collectivités canadiennes (Cycle 2.1). Fichier de microdonnées à grande diffusion, 2003.

12. Starkes JM, Poulin CC, Kisely SR. Unmet need for the treatment of depression in Atlantic Canada. Can J Psychiatry 2005 Sep;50(10):580-90.

13. Robins LN, Wing J, Wittchen HU, Helzer JE, Babor TF, Burke J, et coll. The Composite International Diagnostic Interview. An epidemiologic instrument suitable for use in conjunction with different diagnostic systems and in different cultures. Arch Gen Psychiatry 1998;45:10069-77.

14. Wittchen HU. Reliability and validity studies of the WHO-Composite International Diagnostic Interview (CIDI): A critical review. J Psychiatr Res 1994;28:57-84.

15. Ohinmaa A, Schopflocher D, Jacobs P, Golmohammadi, K, Demeter, S, Klarenbach, S. A population-based analysis of health behaviors, chronic diseases, and associated costs. Chronic Dis Can 2006;27(1):17-24.

16. Institut canadien d'information sur la santé. Rapport sur les catégories du système de groupement national, Canada 2002-
2003. Ottawa : Institut canadien d'information sur la santé, 2005.

17. Feeny D, Furlong W, Boyle M, Torrance GW. Multi-attribute health status classification systems: Health utilities index. Pharmacoeconomics 1995;7:490-502.

18. Feeny D, Furlong W, Torrance GW, et coll. Multi-attribute and single-attribute utility functions for the Heath Utility Index Mark 3 System. Med Care 2002;40(2):113-128.

19. Hirth RA, Chernew ME, Miller E, Fendrick AM, Weissert WG. Willingness to pay for a quality-adjusted life year: In search of a standard. Med Decis Making 2000; 20(3):332-42.

20. Sareen, J, Stein MB, Campell DW, Hassard $\mathrm{T}$, Menec $\mathrm{V}$. The relation between perceived need for mental health treatment, DSM diagnosis, and quality of life: A Canadian population-based survey. Can J Psychiatry 2005;50(2):87-94.

21. Laupacis A, Feeny D, Detsky AS, Tugwell PX. How attractive does a new technology have to be to warrant adoption and utilization? Tentative guidelines for using clinical and economic evaluations. CMAJ 1992;146(4):473-81.

22. Hutubessy RC, Van Tulder MW, Vondeling H. Annu Meet Int Soc Technol Assess Health Care. Int Soc Technol Assess Health Care Meet 1996;12:40.

23. Kessler RC, Ormel J, Demler O, et coll. Comorbid mental disorders account for the role impairment of commonly occurring chronic physical disorders: Results from the National Comorbidity Survey. J Occup Environ Med 2003;45:1257-66.

24. Kessler RC, Frank RG. The impact of psychiatric disorders on work loss days. Psychol Med 1997;27:861-73.

25. Durbin J, Rush B, Smith B, Thibault K. The Impact Study Mid-Term Report 2007. Toronto : Health Systems Research and Consulting Unit, Centre for Addiction and Mental Health, 2007. 